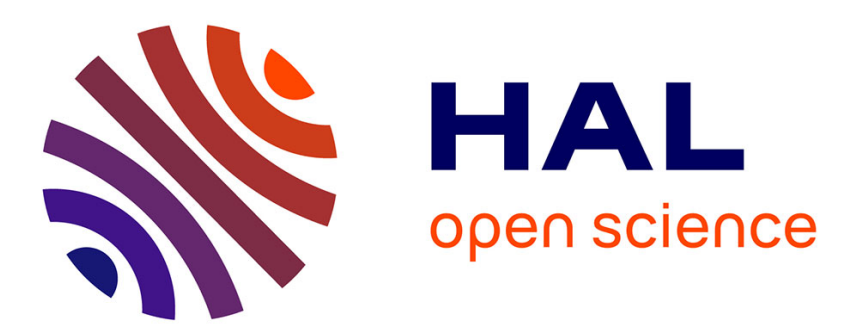

\title{
Efficient self-control device based on inspection model for Pitot sensors system
}

\author{
Wajih Ezzeddine, Jérémie Schutz, Nidhal Rezg
}

\section{To cite this version:}

Wajih Ezzeddine, Jérémie Schutz, Nidhal Rezg. Efficient self-control device based on inspection model for Pitot sensors system. Engineering Failure Analysis, 2019, 103, pp.400-409. 10.1016/j.engfailanal.2019.04.017 . hal-02133829

\section{HAL Id: hal-02133829 \\ https://hal.univ-lorraine.fr/hal-02133829}

Submitted on 25 Oct 2021

HAL is a multi-disciplinary open access archive for the deposit and dissemination of scientific research documents, whether they are published or not. The documents may come from teaching and research institutions in France or abroad, or from public or private research centers.
L'archive ouverte pluridisciplinaire HAL, est destinée au dépôt et à la diffusion de documents scientifiques de niveau recherche, publiés ou non, émanant des établissements d'enseignement et de recherche français ou étrangers, des laboratoires publics ou privés.

\section{다)(1) $(5$}

Distributed under a Creative Commons Attribution - NonCommerciall 4.0 International 


\title{
Efficient self-control device based on inspection model for Pitot sensors system
}

\author{
Wajih Ezzeddine, ${ }^{\mathrm{a},}$, Jeremie Schutz ${ }^{\mathrm{a}}$, Nidhal Rezga \\ ${ }^{a}$ LGIPM, Universit de Lorraine, Metz, France
}

\begin{abstract}
This paper proposes a new inspection model for a multi-component system, subject to a deterioration process which impacts its efficiency. To control the deterioration process, several inspections are carried out after which various decisions are made on how to evaluate the functioning of the system. The proposed inspection model is applied on the case of Pitot sensors system for aircraft. This study is derived from an aeronautic problem defined following the crash of the Airbus 330 in the Rio-Paris flight. Since the Pitot sensors system is non-self-announcing, the paper proposes a technical solution which consists in setting up an additional self-control device in order to inspect and calibrate the already installed Pitot sensors measurements. The objective of this paper is to determine an optimal inspection policy which minimizes the total measurement quality degradation per unit of time in a finite time horizon. Numerical examples and a comparative study are provided to illustrate the proposed analytic model and to highlight the contribution of the paper.
\end{abstract}

Keywords: self-control, degradation, Pitot sensors, optimal inspection, measurement quality

\section{Introduction}

The growing importance of maintenance leads to an increasing interest in the implementation of inspection models for deteriorating systems. Different researches have produced many interesting and significant results for a huge variation of inspection models. The existing models in the literature depend on the assumptions regarding, for example, the time horizon, the nature of cost

\footnotetext{
* I am corresponding author

Email addresses: wajih.ezzeddine@univ-lorraine.fr (Wajih Ezzeddine). jeremie.schutz@univ-lorraine.fr (Jeremie Schutz), nidhal.rezg@univ-lorraine.fr (Nidhal Rezg)
} 
functions, the objective of the models, and so on.

Many attempts to study continuously deteriorating systems have been made under simplifying assumptions. Markov and semi-Markov models are the principal approaches in modeling conditional maintenance. Grall et al. (2002) develop a mathematical model in order to assess the performance of the proposed maintenance policy and to minimize the long run expected maintenance costs per unit of time. Berenguer et al. (1997) consider a stochastically continuous deteriorating non-self-announcing system. The proposed maintenance policy aims to minimize the long-run average cost incurred by inspections, preventive maintenance actions and random breakdowns.

Models dealing with inspection policies may be classified according to the system's operating context such as the inspection costs, the performance criteria, the time span and all constraints related to operating conditions and resource a vailability. Nakagawa (1986) considers a modified inspection policy with periodic check intervals. The model derives the mean time to failure and the expected number of inspections before failure and aims to reduce the total expected cost until detection of failure.

The majority of the models appeared in the literature assume that the system degradation level can only be known through periodic inspection as typical of safety systems of nuclear plants. A common feature of the models discussed is that the state of the system is described as a state of Markov process and then the analysis proceeds to nding analytically the probabilities of the various states [Hontelez et al.(1996), Kopnov (1999)]. However, if the system is made of several multi-state components, the analysis becomes excessively complicated [Barata et al. (2002)]. Many proposals deal with system availability optimization [Chelbi et al. (2008), Badia et al. (2002)]. Sarkar and Sarkar (2000) express the system availability function as well as the limit average availability in order to determine the inspection period. In Ait-Kadiand Chelbi (2010), the suggested inspection strategy aims to reduce the frequency of the random failures and to increase the system availability. Maintenance models have been studied under several constraints and different production systems such as the series-parallel systems in Bris et al. (2003) and multiple product model with single machine in Taleizadeh et al. (2011).

For more details about inspection and maintenance models, the reader may refer to the review of literature in Chelbi and Ait-Kadi (2009).

To resolve maintenance and inspection policies, different heuristic and metha-heuristic 
methods were carried out from which we cite methods derived from genetic algorithms (GA). GA have been promoted as a class of generalpurpose search strategies that maneuver through complex space in a near optimal way. Genetic algorithms can be viewed as a class of optimization methods based on natural principles, i.e. evolution and selection. The main key of a Genetic Algorithm is to maintain a set of candidate solutions, called populations. Members of the population are usually represented as strings, referred to as chromosomes, and evaluated according to a fitness function. Bjarnason (2014) proposes the GA method to find the combination of decision variables in order to minimize the total cost over the system life cycle in the simulation model. Lapa et al. (2006) propose GA as an appropriate optimization technique to deal with the problem of a preventive maintenance policy evaluation based upon a cost-reliability model.

Production systems of goods and services are generally subject to continuous deterioration process with respect to both age and usage. Such a deterioration impacts directly the system performances and production quality. As an example, many plane crashes were due to the Pitot system deterioration. For instance, the Airbus A330 plane, operated by Air France, from Rio de Janeiro to Paris, which crashed into the Atlantic Ocean, killing all 228 passengers aboard the aircraft. BEA's “Bureau d'Enquetes et d'Analyses (BEA) pour la Securite de l'Aviation civile" final report concluded that the aircraft crashed after temporary inconsistencies between the airspeed measurements given by the Pitot sensors system, after which the crew reacted incorrectly and ultimately led the aircraft to crash into the ocean.

The Pitot sensor reliability and maintainability to provide accurate measurements was the point of interest of many proposals. Cox reliability modeling is currently one of the most used methods to estimate systems reliability functions. The Cox model applications and extensions were investigated in the proposals of Hosmer et al. (1999) and those of Kalbfleish et al. (1980). Recently, Ezzeddine et al (2015) applied the Cox regression process to express the Pitot probe reliability as a function of parameters which are assumed to be affecting the good functioning of the sensors. Ezzeddine et al (2016a) studied the extension of Cox modelling to investigate the case of time-dependent covariates. However, no significant research work dealed with the problem of increasing the Pitot sensors systems accuracy during a flight despite of the system importance and the practical need to reduce accidents linked to Pitot sensors measurement inaccuracy and incoherence.

To avoid such critical situations and accidents, the current study proposes to as sess the 
Pitot sensors system measurements degradation during the flight. The Pitot sensors system is a non-self-announcing equipment whose the measurement degradation level can be only determined through inspection. Each inspection cons is ts in assessing one or several characteristics to evaluate the degradation level. An inspection strategy establishes the dates at which one or more operating parameters have to be checked, in order to determine the state of the system. In addition to that, a technical solution might be to add a device to inspect sensors degradation. the device frequency of checks will be the subject of optimization in this paper. The paper is structured as following:

Section 2 describes the proposed additional device for Pitot sensors measurements inspection.

Section 3 gives the mathematical variables that will be used in the paper for inspection policies modelling.

Section 4 details the measurement degradation model for the Pitot sensors (Fix Pitot sensors) and for the additional device.

Section 5 and Section 6 describe the two inspection models: Periodic inspection model with fix intervals and sequential inspection model with varied intervals.

Section 7 provides numerical examples and a comparative study to illustrate the proposed policies.

\section{Device for velocity self-control}

In order to improve the velocity measurement and to avoid accidents linked to Pitot sensors, the project team has design a technical solution based on redundancy concept. The solution consists in adding a device for self-control sensors measurement degradation since the Pitot system is a non-self announcing system. The main objective is to auto-check the Pitot sensors measurements accuracy during the flight. Check frequency will be the subject of optimization in this study. The studied system is composed of a set of fix sensors which are permanently out in order to give instantaneously, during the flight, different information about the pressure and the speed of the air and an additional device which inspects the measurements given by the fix sensors and on which the optimal inspection policy is based. In the current study, this additional device will be modeled by a retractable sensor. This sensor has a different work cycle compared to fix sensors:

- When the Fix sensors work well, the retractable sensor is permanently inward and retracts 
outward only at the inspection dates.

- If the Fix sensors fail, the retractable sensor is permanently outward and behaves as a Fix sensor.

Thus, on the software side, two aspects need to be investigated:

- The retractable sensor measurement value is considered as a reference while it's degradation didn't reach a given threshold.

- The retractable sensor, estimates each fix tube measurement quality degradation.

The degradation process of this device is given in details in the section 5 .

The aim of this work is to optimize the inspection frequency in function of velocity measurement quality degradation. In this case, after each inspection, different decisions could be made on the basis of the measurement quality degradation level of each component. The term degradation corresponds to the sensors measurement quality degradation in function of time. The first task consists in computing different measurements given by each sensor and then decide about the weight of its measurement. The second task concerns the length of the period between two successive inspections in order to get the optimal performances. The system should not be often inspected because each inspection might degrade the used device during the studied interval.

\section{Notations}

- $T_{\text {miss }}$ : finite time horizon (flight duration), expressed in minutes in the illustration examples;

- $\quad H$ : number of time steps in $T_{\text {miss }}$;

- $t$ : time step, expressed in minutes in the illustration examples. The time step is computed as following: $\mathrm{t}=T_{\text {miss }} / H$

- $\quad N$ : number of Fix sensors;

- $r_{i}(0)$ : initial degradation level for the sensor $i$ (at $t=0$ );

- $r_{i}(t)$ : degradation level at the time $t$ for the Pitot tube $i(i=1, \ldots N)$;

- $\quad r_{m}$ : first degradation threshold;

- $\quad r_{\max }$ : second degradation threshold $\left(r_{\max } \geq r_{m}\right)$; 
- $\quad D e g_{\text {ret }}$ : degradation threshold of the retractable sensor at which its measurement is not reference anymore.

- $\quad N c$ : number of expected inspections;

- $\quad N c^{*}$ : optimal number of inspections

- $T^{*}$ : optimal inspection period. In the case of periodic inspection, $T^{*}=T_{\text {miss }} / N c^{*}$. In the case of sequential inspection, $T^{*}=\left(T_{1}^{*}, T_{2}^{*}, \ldots T_{N c}^{*}\right)$ corresponds to the vector of optimal inspection dates.

- $\quad \mu_{t}:$ total expected measurement quality degradation;

- $\mu_{s}$ : degradation per time unit of the retractable sensor at each inspection;

- $\quad \mu_{1}$ : degradation per time unit of a sensor from the date at which it reaches $r_{m}$ to the next inspection date;

- $\mu_{2}$ : degradation per time unit of a sensor from the date at which it reaches $r_{\max }$ to the next inspection date;

- $\tau_{1, i}$ : first date at which the degradation $r_{m}$ is exceeded (Figure 1);

- $\tau_{2, i}$ : first date at which the degradation $r_{\max }$ is exceeded (Figure 1).

\section{Assumptions For the Degradation Model}

\subsection{Fix Pitot Sensor}

During the flight, the Pitot sensor is non-repairable. The sensor measurement quality degradation in time is assumed to be random. In addition, the following assumptions are considered:

- the sensor $i$ is designed to be functional during a defined finite time horizon $T_{\text {miss }}$; this finite duration is divided into successive time steps;

- each sensor $i$ has a specified initial degradation level, noted $r_{i}(0)$;

- the sensor degradation level during a defined mission cannot decrease;

- there is a maximum allowed degradation level $r_{\max }$, which the sensor $i$ may reach within its mission time;

- if the $i^{\text {th }}$ component reaches a given degradation threshold $r_{m}$, a less weight has to be 
awarded to its measurements values compared to other tubes.

- if at any given time $t<T_{\text {miss }}, r_{i}(t)>r_{\max }$ the component $i$ is considered as a defective equipment.

- the Pitot sensor degradation process is expressed as follows:

$$
r_{i}(t)=r_{i}(t-1)+\psi
$$

With $\psi \geq 0$ a random degradation level increase between $t-1$ and $t$.

\subsection{Retractable Pitot Sensor}

As mentionned in the section 2, the retractable sensor corresponds to the device which will be used to inspect the Fix tubes measurement accuracy and on which the proposed inspection policies are based. The following assumptions are considered for Retractable tube degradation model:

- the component is designed to be functional for a defined mission $T_{\text {miss }}$;

- When Fix Pitot sensors work well, the retractable tube degrades by a fixed quantity at each inspection. The number of inspections will be subject to optimization in the proposed inspection polices.

\subsection{Multiple non-repairable system}

Consider the whole system components (multicomponent non-repairable system) which components are inspected periodically in order to detect as soon as possible failure occurrence. Specific assumptions are given below:

- the system is made of $N$ Fix Pitot sensors and one retractable tube;

- failures are assumed to break the components. Thus, in case of failure at time $t, a$ component cannot be repaired and has to be ignored during the mission remaining time.

- In the following sections, the system is assumed to be subject to random deterioration as described in the presented degradation model

\section{Periodic inspection}

\subsection{Periodic Inspection Model}

This section deals with an inspection policy with periodic check intervals (Figure 2). The main 
objective is to minimize the total expected measurements quality degradation during a given mission. The proposed policy is investigated in a numerical example.

At each time step, one of the following events takes place exclusively for each sensor of the system:

- Sensor $i$ reaches $r_{m}$, respectively $r_{\max }$ : The system measurement quality degrades as quickly as it is not yet inspected. This will be expres sed by an additional quantity of quality degradation increased for each unit of time between the times at which $r_{m}$, respectively $r_{\max }$ is exceeded and its inspection date (Figure 3).

- None of the above: sensor $i$ continues its normal operation;

The optimization problem of this section regards the choice of the optimal periodic inspection policy which allows to assess the $N$ component degradation as soon as possible and as a consequence maximize the system measurements accuracy. The periodicity is optimized with respect to the degradation of the retractable sensor. The objective is then to minimize the expected total system measurements quality degradation during the operating mission time. In order to reach this objective, we propose a degradation function which expresses the total degradation of the system measurements quality.

We consider $\Delta_{m, k}^{i}$ and $\Delta_{\max , k}^{i}$ used respectively to identify the time step when the date at which the degradation $r_{m}$ and $r_{\max }$ are exceeded. These two variables are expressed as following:

$$
\begin{aligned}
\text { - } & \Delta_{m, k}^{i}= \begin{cases}1 & \text { if }(k-1) T<\tau_{1, i}<k T \\
0 \text { otherwise }\end{cases} \\
\text { - } \Delta_{\max , k}^{i} & = \begin{cases}1 & \text { if }(k-1) T<\tau_{2, i}<k T \\
0 \text { otherwise } & \end{cases}
\end{aligned}
$$

The total of the degradation values in the system is given by:

$$
\begin{aligned}
\mu_{t}=N c \cdot \mu_{s} & +\sum_{i=1}^{N} \sum_{k=1}^{N c} \mu_{1} \cdot\left(k T-\tau_{1, i}\right) \cdot \Delta_{m, k}^{i}+\sum_{i=1}^{N} \sum_{k=1}^{N c} \mu_{2} \cdot\left(k T-\tau_{2, i}\right) \cdot \Delta_{\max , k}^{i} \\
& -\sum_{i=1}^{N} \sum_{k=1}^{N c} \mu_{1} \cdot\left(k T-\tau_{2, i}\right) \cdot \Delta_{m, k}^{i} \cdot \Delta_{\max , k}^{i}
\end{aligned}
$$

The objective of this model is to minimize the total of the degradation values in the system while the degradation of the retractable sensor $N c \cdot \mu_{s} \leq \operatorname{Deg}_{\text {ret }}$.

$\tau_{1, i}$ and $\tau_{2, i}$ are evaluated on the basis of the equation (1) which gives the Pitot sensor 
degradation process in function of time.

In the equation (2)

- $N c \cdot \mu_{s}$ : the first term expresses the degradation of the device (retractable sensor) at each inspection, this term is proportional to the number of realized inspections.

- $\quad \sum_{i=1}^{N} \sum_{k=1}^{N c} \mu_{1} \cdot\left(k T-\tau_{1, i}\right) \cdot \Delta_{m, k}^{i}$ : This term expresses the measurement quality degradation due to the delay of the non-inspection of the reaching of the first $r_{m, i}$

- $\quad \sum_{i=1}^{N} \sum_{k=1}^{N c} \mu_{2} \cdot\left(k T-\tau_{2, i}\right) \cdot \Delta_{\text {max }, k}^{i}$ :This term expresses the measurement quality degradation due to the delay of the non-inspection of the reaching of the first $r_{\max , i}$

- $\quad \sum_{i=1}^{N} \sum_{k=1}^{N c} \mu_{1} \cdot\left(k T-\tau_{2, i}\right) \cdot \Delta_{m, k}^{i} \cdot \Delta_{\text {max }, k}^{i}$ this part of the model is to eliminate the effect of $\mu_{1}$ during $\tau_{2, i}$ since it is overlapped with $\mu_{2}$ at the same period, $\tau_{2, i}$.

\subsection{Solving procedure}

Dealing with the periodic inspection problem, the optimal solution is provided by the algorithm illustrated in Figure 4. The method consists in computing the total degradation quantity for each possible number of inspections and choosing the minimum. The total number of inspections $N c$ is bounded between 1 and the number of the time horizon steps given by $H$.

\section{Sequential Inspection}

\subsection{Sequential Inspection Model}

Stand-by system failures are usually checked during regular inspections. The periodic inspection policy is usually the most adopted one. However, during the inspection, a lot of information is obtained about the status of the system degradation. Such information should be used to decide for the next inspection date. Hence sequential inspection is more appropriate, especially in the case of randomly failing systems, where the status of component degradation has to be estimated with the information from inspection (Figure 5).

In this section, a sequential inspection policy $T_{k}^{*}(k=1,2, \ldots N c)$ is proposed to minimize the total degradation rate $\mu_{t}$. Assuming the same assumptions and notations of the previous 
section, the total degradation of the system, in the case of sequential inspection, is expressed as follows:

$$
\begin{aligned}
\mu_{t}=N c \cdot \mu_{s} & +\sum_{i=1}^{N} \sum_{k=1}^{N c} \mu_{1} \cdot\left(T_{k}-\tau_{1, i}\right) \cdot \alpha_{\mathrm{m}, k}^{i}+\sum_{i=1}^{N} \sum_{k=1}^{N c} \mu_{2} \cdot\left(T_{k}-\tau_{2, i}\right) \cdot \alpha_{\max , k}^{i} \\
& -\sum_{i=1}^{N} \sum_{k=1}^{N c} \mu_{1} \cdot\left(T_{k}-\tau_{2, i}\right) \cdot \alpha_{\mathrm{m}, k}^{i} \cdot \alpha_{\max , k}^{i}
\end{aligned}
$$

Where: $\alpha_{m, k}^{i}$ and $\alpha_{\max , k}^{i}$ used respectively to identify the time step when the date at which the degradation $r_{m}$ and $r_{\max }$ are exceeded. These two variables are expressed as following:

$$
\begin{aligned}
\text { - } \alpha_{m, k}^{i} & = \begin{cases}1 & \text { if } T_{k-1}<\tau_{1, i}<T_{k} \\
0 \text { otherwise }\end{cases} \\
\text { - } \alpha_{\max , k}^{i} & = \begin{cases}1 & \text { if } T_{k-1}<\tau_{2, i}<T_{k} \\
0 \text { otherwise }\end{cases}
\end{aligned}
$$

Dealing with the present sequential inspection problem, the solution is provided by a process based on a genetic algorithm. Figure 6 illustrates the flowchart of the proposed algorithm. According to this flowchart, the first step corresponds to the initialization where input data are provided.

The second step consists in computing, for each iteration, the optimal solution from those generated by the genetic algorithm.

Where $I T_{\max }$ is the maximum number of iterations. It designs the stopping criterion of the genetic algorithm. Once this number is reached, the algorithm stops and prints the optimal solution. The choice of this number depends on the algorithm, the population size and the problem complexity.

\subsection{Genetic Algorithm Application for sequential inspection model}

\subsubsection{Coding}

In the current case, each individual presents a candidate solution for the optimal sequential policy problem. The candidate solution is an array of $\mathrm{H}$ bits. These bits present the chromosomes which compose individuals and have binary values. When chromosome $\mathrm{i}$ is evaluated at 1 , which means that an inspection is realized at the date $\mathrm{i}$ and 0 otherwise. Each solution proposes a set of inspection dates. 
The following sequence presents a coding for an individual associated with the determination of a sequential inspection policy.

\begin{tabular}{|c|c|c|c|c|c|c|c|}
\hline 0 & 1 & 0 & 0 & 1 & 0 & 0 & 1 \\
\hline$\downarrow$ & $\downarrow$ & $\downarrow$ & $\downarrow$ & $\downarrow$ & $\downarrow$ & $\downarrow$ & $\downarrow$ \\
\hline$T_{1}$ & $T_{2}$ & $T_{3}$ & $\ldots \ldots \ldots \ldots \ldots \ldots \ldots \ldots \ldots \ldots \ldots \ldots \ldots \ldots \ldots \ldots \ldots \ldots \ldots \ldots \ldots \ldots \ldots \ldots \ldots \ldots \ldots$ & $T_{H}$ \\
\hline
\end{tabular}

In this example, the first inspection is performed at the end of the second step of time, and the last one at the end of the $H^{\text {th }}$ period.

A group of candidate solutions is called a population and a group of populations constitutes a generation.

\subsubsection{Reproduction Strategy}

To form a new generation, some individuals have to be selected in each iteration to be modified. The selection process is based on a competition between individuals (called parents) in order to select the best individuals deserving to reproduce the new generation. For each competition, individual having the best fitting participate in the production of new individuals (children) through different mechanisms of crossover and mutation. In this case, fitness correspond s to the total degradation rate associated to the solution provided by the concerned individual.

\subsubsection{Crossover Operator}

There are many ways to apply the crossover operator. In this case, the Crossover operator at the individual level is chosen. A crossover point is randomly chosen for two randomly selected individuals (parents). This point occurs between two bits and divides each individual into left and right sections. Crossover then swaps the left or the right selection of the two individuals. As an example of crossover, consider the two parents:

Parent 1:1010101011

Parent 2:1000010001

Suppose the crossover point randomly occurs after the fourth bit. Then new childs are presented as following: 
Child 1:1010010001

Child 2:1000101011

\subsubsection{Mutation Operator}

This operator consists of alerting one or more gene values in a chromosome from its initial value. It is analogous to the biological mutation. In this case, the flip-bit mutation is applied. This mutation operator takes the chosen chromosome and inverts a randomly bit value.

Parent 1: 0001001001

Child 1: 0011001001

The chromosome subject to value changing is chosen randomly.

\subsubsection{Constraints}

In this case, it is assumed that the Pitot system has to be inspected at least for the last time step of the studied finite horizon. So, in the solutions generation, the genetic program is forced to attribute 1 to the chromosome which corresponds to the final time step (i.e. $T_{H}=1$ ).

To ensure an individual is valid, which means that the generated individual verifies the constraints of the model, a test should be realized. This test has to be carried out for every new individual after its production through crossover and mutation operators.

\section{Numerical examples and comparatives studies}

In this section, several numerical examples dealing with the period ic inspection, the sequential one and a comparative studies between these two policies and other studies are illustrated.

\subsection{Periodic inspection policy}

The first numerical example is proposed to illustrate the first investigated inspection model. It is assumed that different quantities of degradations are well estimated and known. Furthermore, probability distributions of system degradation composed of three components and time to failure are assumed to be well estimated. In the present numerical example, these input parameters are 
given by:

- $\quad T_{\text {miss }}=100$ minutes

- $\quad H: 20$ periods

- $\mu_{s}=0.2$

- $\mu_{1}=0.05$

- $\mu_{2}=0.1$

- $r_{1}(0)=0.1$

- $r_{2}(0)=0.05$

- $r_{3}(0)=0$

- $\quad r_{m}=0.3$

- $r_{\max }=0.7$

- $\quad$ Deg $_{\text {ret }}=0.8$

As mentioned before, for each Pitot tube $i$, the degradation process is expressed by:

$$
r_{i}(t)=r_{i}(t-1)+\psi
$$

Where

- $\tau_{1, i}=\inf \left\{t / r_{i}(t) \geq r_{m}\right\}$

- $\tau_{2, i}=\inf \left\{t / r_{i}(t) \geq r_{\max }\right\}$

$\psi$ is a random positive variable, in this example, the $\psi$ values are generated as given in Table 1:

Table 1: $\psi$ values at each time step

\begin{tabular}{|l|c|c|c|}
\hline & Sensor 1 & Sensor 2 & Sensor 3 \\
\hline 1 & 0.05 & 0.05 & 0 \\
\hline 2 & 0 & 0 & 0.05 \\
\hline 3 & 0.05 & 0.025 & 0 \\
\hline 4 & 0.025 & 0.05 & 0.05 \\
\hline 5 & 0.05 & 0.025 & 0.05 \\
\hline 6 & 0 & 0 & 0 \\
\hline
\end{tabular}




\begin{tabular}{|l|c|c|c|}
\hline 7 & 0.025 & 0.05 & 0.05 \\
\hline 8 & 0.05 & 0 & 0.025 \\
\hline 9 & 0.1 & 0.05 & 0.05 \\
\hline 10 & 0.05 & 0.1 & 0 \\
\hline 11 & 0.1 & 0.025 & 0.025 \\
\hline 12 & 0.025 & 0.05 & 0.025 \\
\hline 13 & 0.025 & 0 & 0 \\
\hline 14 & 0.05 & 0.1 & 0.1 \\
\hline 15 & 0.025 & 0.025 & 0.05 \\
\hline 16 & 0.05 & 0.05 & 0.025 \\
\hline 17 & 0.1 & 0.05 & 0.025 \\
\hline 18 & 0.025 & 0.025 & 0.05 \\
\hline 19 & 0.1 & 0 & 0 \\
\hline 20 & 0 & 0.1 & 0.1 \\
\hline
\end{tabular}

Thanks to the application of the algorithm of the figure 4, the optimal periodic inspection policy corresponds to $\mathrm{Nc}^{*}=4 ; \mathrm{T}^{*}=100 / 4=25$ minutes.

\subsection{Sequential inspection policy}

The genetic algorithm parameters are adjusted after assessing the algorithm's performances on a few trial runs. Therefore, these values will be considered:

- Crossover probability $=0.5$

- Mutation probability $=0.01$

- $\quad I T_{\max }=100$

Based on the same data used to illustrate the optimal periodic policy, the following optimal sequence is obtained:

\begin{tabular}{|l|l|l|l|l|l|l|l|l|l|l|l|l|l|l|l|l|l|l|l|}
\hline 0 & 0 & 0 & 0 & 0 & 0 & 0 & 0 & 1 & 0 & 0 & 0 & 0 & 1 & 0 & 0 & 1 & 0 & 0 & 1 \\
\hline
\end{tabular}

The obtained sequence means that inspection dates are respectively 9,14, 17 and 20.

The total degradation corresponding to this optimal sequence is: $\mu_{t}=1.05$. 
The optimal number of inspections is $N c^{*}=4$.

Table 2 shows the influence of the amounts of quality degradation on the optimum check times:

Table 2: Optimum sequential inspection dates which minimize the total degradation quantity

\begin{tabular}{|c|c|c|c|c|c|c|c|c|c|c|c|c|c|c|c|c|c|c|c|c|c|c|}
\hline$\left(\mu_{1}, \mu_{2}\right)$ & \multicolumn{20}{|c|}{ Optimal sequence } & \multirow{2}{*}{$\begin{array}{c}N c^{*} \\
3\end{array}$} & \multirow{2}{*}{$\begin{array}{c}\mu_{t} \\
0.81\end{array}$} \\
\hline$(0.01,0.05)$ & 0 & 0 & 0 & 0 & 0 & 0 & 0 & 0 & 0 & 0 & 1 & 0 & 0 & 1 & 0 & 0 & 0 & 0 & 0 & 1 & & \\
\hline$(0.05,0.1)$ & 0 & 0 & 0 & 0 & 0 & 0 & 0 & 0 & 1 & 0 & 0 & 0 & $\overline{0}$ & 1 & 0 & 0 & 1 & 0 & $\overline{0}$ & 1 & 4 & 1.05 \\
\hline$(0.15,0.2)$ & $\overline{0}$ & 0 & 0 & 0 & 0 & 0 & 1 & 0 & 1 & 0 & 1 & 0 & 0 & 1 & 0 & 0 & 1 & 0 & 0 & 1 & 6 & 1.2 \\
\hline
\end{tabular}

This experiment investigates the case where the inspection policy is sequential. The measurements quality degradation quantities are made variable. For different values of $\left(\mu_{1}, \mu_{2}\right)$, Table 2 gives the optimal vector of inspection dates. For each value of the optimal vector, Table 2 also gives the optimal average total system degradation.

\subsection{Comparative study: Periodic vs. sequential inspection}

The aim of this section consists to compare the periodic and sequential inspection policies.

Based on the numerical examples results, the optimal number of inspections is the same for the two different policies $N c^{*}=4$. On the basis of the same inputs, the optimal average total cost per time unit induced by the measurement quality degradation has decreased from 1.6 for the periodic policy to 1.05 for the sequential one.

\section{Proposition 1:}

The sequential inspection policy is preferred to the periodic one and it is more optimal.

\section{Discussion:}

The equations (2) and (3) show clearly that the periodic inspection model is just a specific case for the sequential one. In the periodic model, we have one constraint more compared to the sequential policy (the period between dates of inspections has to be the same). Thus, it can be concluded that the optimal solution given by the sequential policy is equal or better than the optimal one given by the periodic inspection policy. 


\section{Proposition 2:}

The computation of the optimal sequential policy may take longer than the computation of the optimal periodic one.

\section{Discussion:}

The maximum number of iterations corresponding to the computation of the optimal periodic policy corresponds to the time horizon steps number $H$. For the sequential inspection policy, the number of iterations corresponding to the genetic algorithm is $I T_{\max }$ which is the maximum number of iterations. For each iteration, a number of other iterations are executed to generate new solutions and select the best one. Let $\mathrm{F}$ be the number of these iterations. Thus, we have $I T_{\max } * F$ iterations to compute the optimal solution for the sequential policy. Generally, $I T_{\max }$ is chosen to be very important. Thus, it could be concluded that: $I T_{\max } * F>H$. The above analysis in this section should assist deciders in selecting between the two inspection modes.

\subsection{Sensitivity Study with a proposal dealing with optimal inspection policies}

Since, to our knowledge, there is no research works in the literature linked to inspection policies based on efficiency systems degradation, we propose a comparative study between our work and a proposal dealing with inspection policy based on costs as an optimization criteria.

(K. Ito, 2000) considers a periodic inspection policy for a storage system which has to hold a higher reliability. The author proposes an optimal policy which minimize the average cost of inspections and maintenance actions. In this model, the author considers two cost variables, $c_{1}$ which expresses the cost of each action inspection and $c_{2}$ the total cost of maintenance and inspection actions.

Table 3 resumed the obtained numerical results:

Table 3: Optimal Inspection periods $T^{*}$ which minimize the maintenance and inspection total costs

\begin{tabular}{|c|c|c|}
\hline$c_{1} / c_{2}$ & $T^{*}$ & $N c^{*}$ \\
\hline 0.01 & 201 & 11 \\
\hline 0.05 & 683 & 2 \\
\hline
\end{tabular}




\begin{tabular}{|l|l|l|}
\hline 0.1 & 968 & 1 \\
\hline
\end{tabular}

Where $T^{*}$ and $N^{*}$ express respectively the period between two successive inspections and the number of inspections in a finite time horizon.

For the periodic inspection policy and using the given input data in the section, we obtain results shown in Table 4.

Table 4: Impact of the $\mu_{s}$ variation on the number of inspections.

\begin{tabular}{|c|c|c|}
\hline$\mu_{S}$ & $T^{*}$ (minutes) & $N c^{*}$ \\
\hline 0.5 & 6 & 10 \\
\hline 2 & 25 & 4 \\
\hline 4 & 30 & 2 \\
\hline
\end{tabular}

Numerical results given by (K. Ito, 2000) show that when $c_{1} / c_{2}$ increases, the system should be inspected at longer time intervals. The results show also that number of inspections depends principally on the inspection cost value.

Numerical results given by our proposed periodic inspection policy seem to be in good agreement with the above results. The number of inspections decreases when $\mu_{s}$ increases. On the basis of the comparative study, it seems that our numerical results are in good agreement with works dealing with the same topic.

\section{Conclusion}

In this paper, optimal inspection policies for periodic and sequential cases have been derived. This study is proposed for a multi-unit system which is assumed to be irreparable during its mission. The system is assumed to be subject to random deterioration which impacts its measurement accuracy. The proposed approach aims to assess the degradation in such a way to increase the efficiency and the accuracy of the whole system. To assess this deterioration, an additional efficient device is introduced to perform inspections, after which a decision is made on how to compute system measurements. According less importance to measurements given by a component occurs whenever the level of its deterioration reaches a specific threshold. The 
objective is to determine an optimal inspection policy which minimizes the average total degradation per time unit in a finite time horizon. The current study is applied to the specific case of Pitot sensors system. Numerical examples and computation algorithms are provided to illustrate the proposed inspection models. A comparison of the periodic and sequential inspection policies is also investigated.

As a future work, the developed approach could be generalized to other non-reparable multiple-unit system subject to random deterioration.

\section{References}

[1] Ait-Kadi, D. \& Chelbi, A. (2010). Inspection strategy for availability improvement. Journal of Intelligent Manufacturing, 21(2), 231-235.

[2] Badia, F., Berrade, M.D., \& Campos, C.A. (2002). Optimal inspection and preventive maintenance of units with revealed and unrevealed failures. Reliability Engineering \& System Safety, 78(2), 157-163.

[3] Barata, J., Soares, C.G., Marseguerra, M., \& Zio, E. (2002). Simulation modelling of repairable multicomponent deteriorating systems for on condition maintenance optimization. Reliability Engineering \& System Safety, 76(3), 255-264.

[4] Bérenguer, C., Chengbin, C., \& Grall, A. (1997). Inspection and maintenance planning: An application of semi-markov decision processes. Journal of intelligent manufacturing, 467-476.

[5] Bjarnason, E. T. S., Taghipour, S. (2014). Optimizing simultaneously inspection interval and inventory levels (s, S) for a k-out-of-n system. Reliability and Maintainability Symposium (RAMS), (pp. 1-6). IEEE.

[6] Bris, R., Chatelet, E., \& Yalaoui, F. (2003). New method to minimize the preventive maintenance cost of seriesparallel systems. Reliability Engineering \& System Safety, 82(3), 247-255.

[7] Chelbi, A., Ait-Kadi, D., \& Aloui, H. (2008). Optimal inspection and preventive maintenance policy for systems with self-announcing and non-self-announcing failures. Journal of Quality in Maintenance Engineering, 14(1), 34-45.

[8] Chelbi, A. \& Ait-Kadi, D. (2009). Inspection strategies for randomly failing systems. In 
Handbook of maintenance management and engineering, 303-335. Springer.

[9] Ezzeddine, W., Schutz, J., \& Rezg, N. (2015). Cox Regression model applied to Pitot tube Survival Data. 6th International Conference on Industrial Engineering and Systems Management, 168-172, Seville, Spain.

[10] Ezzeddine, W., Schutz, J., \& Rezg, N. (2016a). Test for additive interaction in proportional hazard model applied to pitot sensors reliability and survivability. 7th IFAC Conference on Management and Control of Production and Logistics, Bremen, Germany.

[11] Grall, A., Berenguer, C., \& Dieulle, L. (2002). A condition-based maintenance policy for stochastically deteriorating systems. Reliability Engineering \& System Safety, 167-180.

[12] Hontelez, J.A., Burger, H.H., \& Wijnmalen, D.J. (1996). Optimum condition-based maintenance policies for deteriorating systems with partial information. Reliability Engineering \& System Safety, 51(3), 267-274.

[13] Ito, K., \& Nakagawa, T. (2000). Optimal inspection policies for a storage system with degradation at periodic tests. Mathematical and Computer Modelling, 31(10), 191-195.

[14] Hosmer, D. \& Lemeshow, S. (1999) Applied survival analysis. Regression modeling time to event data, New York.

[15] Kalbfleisch, J. D. \& Ventice, R. L. F. (1980) The Statistical Analysis of Failure time Data. John Wiley and Sons.

[16] Kopnov, V. (1999). Optimal degradation processes control by two-level policies. Reliability Engineering \& System Safety, 66(1), 1-11.

[17] Lapa, C. M. F., Pereira, C. M. N., de Barros, M. P. (2006). A model for preventive maintenance planning by genetic algorithms based in cost and reliability. Reliability Engineering \& System Safety, 91(2), 233-240.

[18] Nakagawa, T. (1986).Periodic and sequential preventive maintenance policies. Journal of Applied Probability, 23, pp. 536-542

[19] Sarkar, J. \& Sarkar, S. (2000). Availability of a period ically inspected system under perfect repair. Journal of Statistical Planning and Inference, 91(1), 77-90.

[20] Taleizadeh, A. A., Sadjadi, S. J., \& Niaki, S. T. A. (2011). Multiproduct EPQ model with single machine, backordering and immediate rework process. European Journal of Industrial Engineering, 5(4), 388-411.

Figure 1: Stochastic degradation for a Fix Pitot sensor 
Figure 2: Finite time $T_{\text {miss }}$ with periodic $k$ intervals $(k=1 ; 2 ; N c)$

Figure 3: Example of measurement quality degradation due to the first Pitot sensor measurement quality degradation

Figure 4: Numerical process to compute the optimal periodic inspection sequence

Figure 5: Finite time $T_{\text {miss }}$ with sequential $k$ intervals $(k=1 ; 2::: N C)$

Figure 6: Proposed algorithm for sequential inspection model 


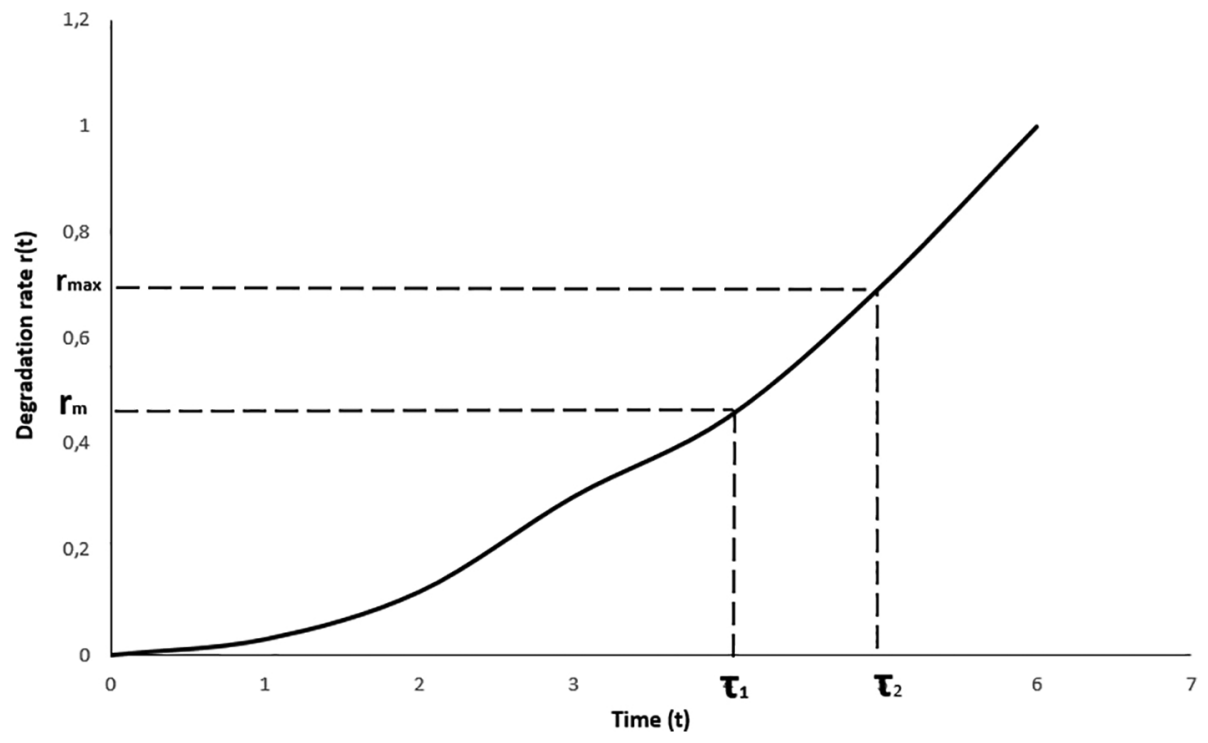

Figure 1 


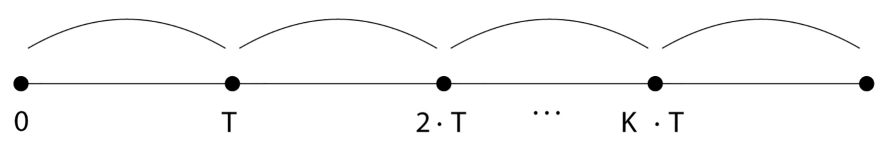

Figure 2 


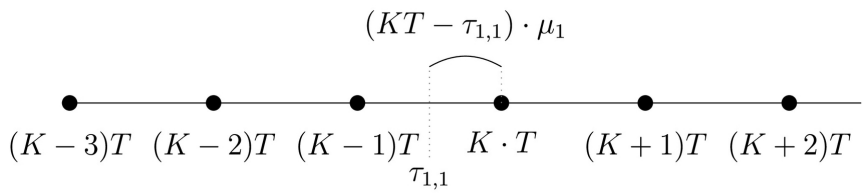

Figure 3 


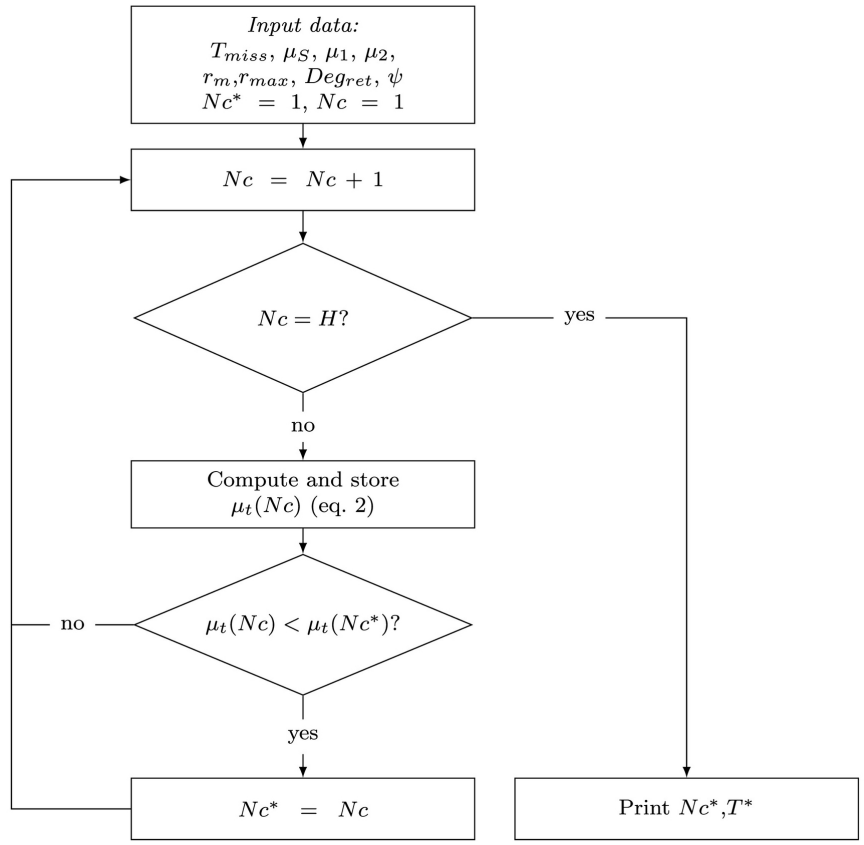

Figure 4 


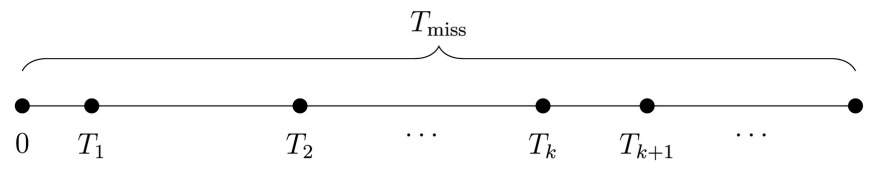

Figure 5 


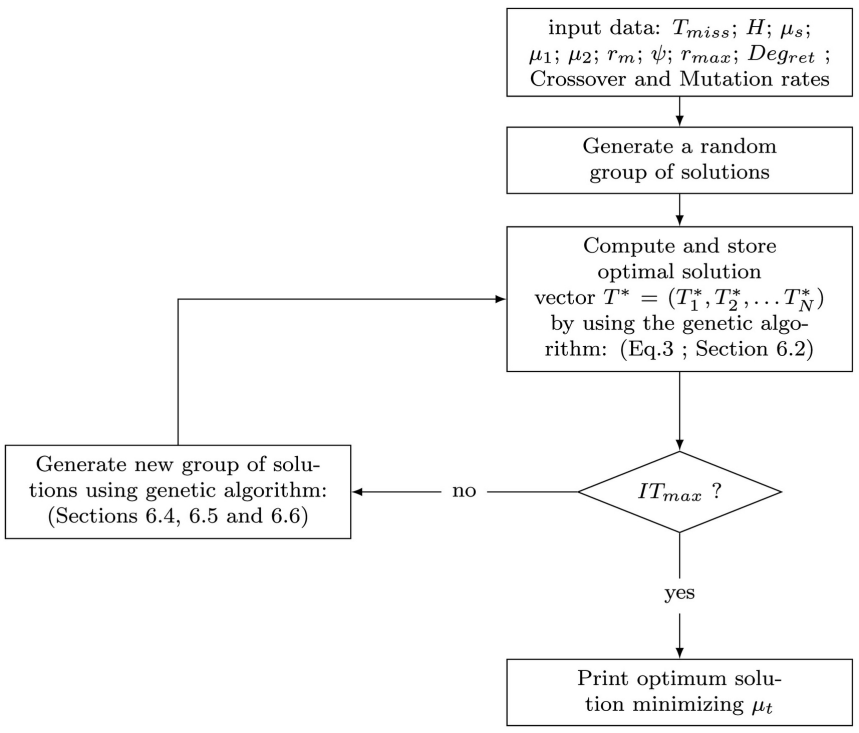

Figure 6 